Como citar este artículo: Giraldo-Ospina B, Henao-Nieto DE, Flórez-Salazar M, Parra-Londoño F, Gómez-Giraldo EL, Mantilla-Moreno OJ. Prevalencia de sífilis en una población de gestantes de dos comunidades de un municipio de Colombia. Revista Biosalud 2015; 14(2): 9-18. DOI: 10.17151/biosa.2015.14.2.2

\title{
PREVALENCIA DE SÍfILIS EN UNA POBLACIÓN DE GESTANTES DE DOS COMUNIDADES DE UN MUNICIPIO DE COLOMBIA ${ }^{1}$
}

\author{
Beatriz Giraldo-Ospina² \\ Daniel Eduardo Henao-Nieto ${ }^{3}$ \\ Mateo Flórez-Salazar ${ }^{4}$ \\ Fernando Parra-Londoño ${ }^{5}$ \\ Ermin Leandro Gómez-Giraldo ${ }^{6}$ \\ Omar Jahir Mantilla-Moreno7
}

\section{RESUMEN}

Objetivo: Estimar la prevalencia de sífilis de una población de gestantes habitantes de las comunidades de La Badea y Frailes del municipio de Dosquebradas (Colombia), intervenidas a través de las prácticas académicas de la Fundación Universitaria Autónoma de la Américas. Metodología: Estudio descriptivo de corte transversal, con muestreo no aleatorio con ajustes por efecto de diseño, de gestantes pertenecientes a un programa de control prenatal. La presencia de sífilis gestacional se determinó mediante pruebas no treponémicas VDRL modificada (Wiener) y pruebas treponémicas (FTA-ABS) confirmatorias para pacientes reactivas. Cada gestante diligenció una encuesta con propósitos de una caracterización social, demográfica y económica. Resultados: De las 101 muestras analizadas, tres gestantes resultaron reactivas a las pruebas no treponémicas $(2,97 \%)$, dos de ellas confirmadas con pruebas treponémicas. La edad promedio fue de 24 años y el promedio de edad gestacional fue de 20 semanas. Se logró demostrar que el antecedente de sífilis es un factor de riesgo asociado a la sífilis gestacional (OR: 2,8 - IC95: 1,8-7,2). Finalmente, se encontraron Infecciones de Transmisión Sexual (ITS) asociadas, se reportaron dos casos de condilomas, dos casos de gonorrea y un caso de herpes genital. Conclusión: Se encontró una prevalencia de $3 \%$ en la población estudiada, la cual se relacionó con el antecedente personal de sífilis. Otras ITS podrían acompañar el diagnóstico de sífilis gestacional, por lo que se recomienda su búsqueda activa ante una paciente con esta enfermedad. No se conoce la real magnitud del problema dado que se presentan múltiples casos de subregistro y subnotificación. La sífilis gestacional aún continúa siendo un problema de salud pública.

Palabras clave: sífilis gestacional, sífilis congénita, comunidades vulnerables.

\footnotetext{
${ }^{1}$ Investigación del Grupo de Investigación en Salud y Comunidad (GISCO) de la Fundación Universitaria Autónoma de las Américas, Pereira, Colombia.

2 Magíster en Microbiología. Profesora, Fundación Universitaria Autónoma de las Américas. Pereira, Colombia. Autor para correspondencia. Correo electrónico: p_giraldob_154@uam.edu.co

${ }^{3}$ Ph.D. en Salud Pública y Epidemiología (C). Profesor, Observatorio EcoRegional de Salud Pública, Facultad de Ciencias de la Salud, Universidad Tecnológica de Pereira. Asesor de la Fundación Universitaria Autónoma de las Américas. Pereira, Colombia. Correo electrónico: d.henao@utp.edu.co

${ }^{4}$ Estudiante X semestre. Facultad de Medicina, Fundación Universitaria Autónoma de las Américas. Pereira, Colombia. Autor para correspondencia. Correo electrónico: salazarmateof@hotmail.com

${ }^{5}$ Estudiante X semestre. Facultad de Medicina, Fundación Universitaria Autónoma de las Américas. Pereira, Colombia. Correo electrónico: ferchoaurora@hotmail.com

${ }^{6}$ Estudiante X semestre. Facultad de Medicina, Fundación Universitaria Autónoma de las Américas. Pereira, Colombia. Correo electrónico: erlean0322@hotmail.com

${ }^{6}$ Estudiante X semestre. Facultad de Medicina, Fundación Universitaria Autónoma de las Américas. Pereira, Colombia. Correo electrónico: tuco8265@hotmail.com
} 


\section{PREVALENCE OF SYPHILIS IN A POPULATION OF PREGNANT WOMEN OF TWO VULNERABLE COMMUNITIES IN A COLOMBIAN MUNICIPALITY}

\section{ABSTRACT}

Objective:To estimate the prevalence of syphilis on a group of pregnant women living in the communities of La Badea and Frailes in the municipality of Dosquebradas (Colombia), intervened through academic practices from Fundación Universitaria Autónoma de las Américas. Methodology: Cross-sectional descriptive study, nonrandom sampling of pregnant women participating in an antenatal control program with adjustments for design effects, The presence of gestational syphilis was determined through modified (Wiener) nontreponemal VDRL tests, and confirmatory treponemal test (FTA-ABS) for reactive patients. Each pregnant woman filled out a survey with the purpose of social, demographic, and economic

\section{INTRODUCCIÓN}

La sífilis, infección de transmisión sexual (ITS) producida por el Treponema pallidum (TP), de distribución mundial, que afecta a 12 millones de personas (1) cada año, tiene una alta tasa de morbilidad y mortalidad. Las manifestaciones clínicas se agrupan cronológicamente en varios periodos, iniciando con la sífilis primaria caracterizada por la formación de una o más lesiones cutáneas (chancros) en el lugar de inoculación de las espiroquetas, las cuales aparecen generalmente a los 21 días a partir del contacto infeccioso. Posteriormente, le sigue la sífilis secundaria que se produce entre la tercera y doceava semanas de aparición del chancro; esta se hace visible por las múltiples manifestaciones cutáneas. characterization. Results: Out of the 101 samples analyzed, 3 pregnant women were reactive to non-treponemal tests $(2.97 \%), 2$ of which were confirmed by treponemal tests. The average age was 24 years and the mean gestational age was 20 weeks. It was possible to demonstrate that the past history of syphilis is a risk factor associated to gestational syphilis (OR: 2,8 - IC95: 1,8-7,2). Finally, associated Sexually Transmitted Diseases (STDs) were found including 2 cases of condyloma, 2 cases of gonorrhea and a case of genital herpes. Conclusion: A prevalence of gestational syphilis of $3 \%$ was found in the studied population which was related with personal history of syphilis. Other STDs would be associated when syphilis by this reason it is recommended an active search in patients with this disease. The actual magnitude of the problem is unknown due to the fact that there are many cases under reported. Gestational syphilis continues to be a public health issue.

Key words: syphilis, pregnancy, congenital syphilis, vulnerable populations.

Posteriormente, la paciente entra en un periodo conocido como latente (2-6), donde las manifestaciones clínicas son escasas y puede alcanzar un periodo de 5 a 50 años como asintomático, tomando especial importancia en las gestantes. La sífilis terciaria ocurre varios años después de la infección, afectando hasta el $40 \%$ de los casos que no reciben tratamiento e incluyendo un amplio espectro de manifestaciones clínicas, siendo las más comunes las complicaciones cardiovasculares, las gomas y las lesiones neurológicas.

La infección por TP es un grave problema cuando ocurre en la embarazada, ocasionando sífilis gestacional (SG), debido a que se transmite fácilmente al feto provocando sífilis congénita (SC) y produciendo -hasta en un $81 \%$ de los casos- complicaciones que incluyen 
hidrops neonatal y restricción del crecimiento intrauterino, prematuridad y bajo peso al nacer, incremento del riesgo de aborto y muerte fetal $(7,8)$ o neonatal temprana, e infección congénita. Por lo tanto, su diagnóstico y tratamiento oportuno se hacen indispensables en todos los casos, estimando que más de dos millones de mujeres embarazadas se infectan con sífilis cada año en el mundo, y un número significativo de casos (692.100 a 1,53 millones) no reciben tratamiento $(7,9-12)$.

La SG se diagnostica en toda mujer embarazada, puérpera o con aborto reciente, úlcera genital o lesiones compatibles con sífilis secundaria y prueba treponémica (incluidas pruebas rápidas) o no treponémica positiva o reactiva, que no ha recibido tratamiento adecuado (antes de la $20^{a}$ semana de gestación y como mínimo 30 días antes del parto) durante la presente gestación. Se considera diagnóstico toda prueba no treponémica (VDRL o RPR) reactiva mayor o igual a 1:8 diluciones o en menores diluciones (1:1, 1:2,1:4) con prueba treponémica (FTA-ABS o TPHA) positiva (13-16).

Recientemente, con el aumento de los casos de infección gestacional y el grave problema de subregistro de la enfermedad -el cual dificulta establecer las dimensiones reales del problema(17), ha surgido la necesidad de implementar estrategias rápidas para la detección y tratamiento (18) en Latinoamérica y el Caribe (LAC), mediante campañas y guías - propuestas por la Organización Mundial de la Salud- para la eliminación de la transmisión maternoinfantil de VIH y SC -indicadores de mal desempeño de sistemas de salud y vigilancia epidemiológica-, a través del tamizaje y el diagnóstico temprano (95\% de las gestantes infectadas), el tratamiento completo y el aumento de la cobertura en control prenatal y parto institucional $(19,20)$, medidas que permitan disminuir la prevalencia de SG a menos del 1\% (21-24) y procuren impactar significativamente la salud materna e infantil, tal como ha sido priorizado en los cuarto, quinto y sexto Objetivos de Desarrollo del Milenio
(4), la disminución de la mortalidad infantil, la mejora de la salud materna y el combate contra VIH/SIDA, la malaria y otras enfermedades, respectivamente (25).

En casos ITS, el manejo sindrómico es aceptado como política de atención en todos los países de la región, si bien, en la mayoría de ellos (15 de 19) el diagnóstico de la sífilis se combina con el manejo etiológico mediante el empleo de VDRL, RPR o pruebas rápidas. En 10 países, las coordinaciones nacionales estiman que se pueden estar atendiendo en el sector informal (farmacias, curanderos, amigos, etc.) entre el 25$50 \%$ de los casos sintomáticos de todas las ITS incluyendo sífilis, y en otros cinco países, aunque se reconoce que este es un problema importante, no se tiene idea de cuál es la proporción que no es atendida en los servicios regulares de salud (26). Este dato puede representar un indicador indirecto del nivel de subregistro existente en la región para las ITS y también de la alta probabilidad de personas que pueden estar recibiendo tratamientos inadecuados y/o incompletos para sus infecciones.

Con excepción de Colombia y Paraguay, todos los países informan la realización de actividades de referencia y control en parejas de casos índices, en quienes informan que 11 de cada 19 $(57,9 \%)$ lo hacen exclusivamente a través de la pareja y otros 4 emplean la estrategia combinada de pareja y proveedor de salud (26).

Según el Informe del evento sífilis gestacional y congénita, en Colombia hasta el periodo epidemiológico 52 del año 2013 (28 de diciembre), se notificaron 20 casos de los cuales 8 corresponden a dicha semana (27). Al analizar la incidencia de sífilis congénita por departamentos, se evidencia que Risaralda ocupa el tercer lugar con mayor número de casos por 1.000 nacidos vivos (28). Con base en lo anteriormente descrito y teniendo en cuenta que las comunidades a intervenir presentan múltiples factores de riesgo y vulnerabilidad, se pretende estimar la prevalencia de sífilis gestacional en 
las gestantes adscritas a un programa de control prenatal de las comunidades La Badea y Frailes, del municipio de Dosquebradas (Risaralda, Colombia).

\section{MATERIALES Y MÉTODOS}

El presente es un estudio de corte transversal para estimar la frecuencia absoluta y relativa de una muestra -suficiente y representativa- de una población de mujeres durante la gestación que asisten a un programa de medicina preventiva (control prenatal), que pretende detectar precozmente cualquier anomalía para tratarla oportunamente y estratificar el riesgo de las gestantes para priorizar la atención en aquellas con riesgo mayor de tener problemas de salud.

El presente estudio se realizó en los sectores de La Badea y Frailes, del municipio de Dosquebradas (Risaralda, Colombia), durante el periodo comprendido entre junio de 2012 y agosto de 2013.

Se tomó como población de interés principal a las gestantes adscritas al programa de control prenatal en los Centros de Salud de dichas comunidades (principal criterio de inclusión). Sin embargo, dada la realidad en el terreno, y las consecuencias desastrosas de omitir un caso de sífilis, todas las mujeres gestantes que quisieran participar del estudio fueron recibidas. En otros casos, se captaron mujeres por medio de demanda inducida, lo cual se refiere al conjunto de actividades encaminadas a universalizar la cobertura de tamización para sífilis en la población gestante de las dos comunas, independiente de su afiliación a un programa de control prenatal.

Dado lo anterior, el proceso de selección de la muestra se realizó mediante un muestreo no probabilístico por conveniencia. Teniendo, que el tamaño de la muestra fue de 101 gestantes, la cual estadísticamente es considerada una muestra significativa y representativa, con la que se esperaba comprobar que en la población cautiva la característica de interés (sífilis gestacional) es de $1 \%$.

Se incluyeron las gestantes que firmaran el consentimiento informado y que vivieran en las comunidades previamente mencionadas, en las cuales se ha realizado un trabajo comunitario previo a través de las prácticas académicas de los estudiantes de la Fundación Universitaria Autónoma de las Américas sede Pereira, a partir del año 2011. Este trabajo, consiste en la formación de estudiantes de Medicina y Odontología alrededor de prácticas académicas cuyo objetivo central es el mejoramiento de la situación de salud a través de un diagnóstico de situación de salud e intervenciones orientadas a impactarlo.

Las gestantes se contactaron por medio de las enfermeras jefes y auxiliares de enfermería de los Centros de Salud de ambas comunidades, las Madres Fami de Hogares Comunitarios de Bienestar FAMI del Instituto Colombiano de Bienestar Familiar, que trabajan en el programa Primera Infancia del ICBF reglamentado en 1989. Estas Madres Fami son las encargadas de atender a las madres gestantes y lactantes, y a los niños hasta los dos años, enseñándoles a las familias prácticas de cuidado y crianza. A este grupo de gestantes se les brindó una charla informativa acerca de la sífilis y sus consecuencias, y posteriormente se les invitó a participar de forma voluntaria en el estudio. Se explicaron los procedimientos a realizar así como los beneficios, en concordancia con la Declaración de Helsinki.

Luego de diligenciar el consentimiento informado, se llevó a cabo una encuesta epidemiológica de 24 preguntas que incluía 12 variables de caracterización sociodemográfica, 9 variables de antecedentes clínicos y bioquímicos y 3 variables de tratamiento. Dentro del instrumento de medición se indagó por variables como asistencia a controles prenatales y antecedentes de ITS, evidencia clínica de sífilis, 
gonorrea y/o condilomas, fecha de última serología y resultado de las pruebas, tratamiento, qué tipo de tratamiento y controles serológicos posteriores.

En estado de ayuno, fueron tomadas 101 muestras de sangre de gestantes adscritas al programa de control prenatal en los Centros de Salud de dichas comunidades, de diferentes edades que aceptaron participar de forma voluntaria en el estudio. Luego de obtener el suero, se realiza el test de VDRL-USR mediante el método de floculación según recomendaciones del fabricante $(13,29)$.

Toda prueba con resultado Reactivo o Reactivo Débil en la fase cualitativa se tituló a partir de 1:1 (suero sin diluir), 1:2, 1:4, 1:8, 1:16, 1:32, 1:64 y así sucesivamente, para reportar hasta el último título de la dilución en que se observó claramente la floculación para informarse cuantitativamente. Según la definición de caso de sífilis gestacional, establecida en el país, se ha determinado la utilización de las pruebas treponémicas en los casos en los que las pruebas no treponémicas, como VDRL-USR, tengan resultados menores de 8 diluciones (13). Estas fueron informadas al Hospital Santa Mónica de Dosquebradas, e independiente del título se confirmaron mediante prueba treponémica de FTA-ABS (inmunofluorescencia indirecta con absorción del suero), quien se encargó de realizar tratamiento y seguimiento, mediante un método de observación directo según recomendaciones del fabricante $(13,30)$.

El estudio se realizó según los lineamientos de la Resolución 008430 de 1993 del Ministerio de Salud y Protección Social de Colombia, y fue avalado por el Comité Científico del Hospital Santa Mónica de Dosquebradas y el Comité de Ética del Departamento de Investigaciones de la Fundación Universitaria Autónoma de las Américas.

Los datos obtenidos en la aplicación de las encuestas, análisis de historias clínicas y resultados del procesamiento de las muestras fueron organizados en tablas electrónicas en el programa Prism 5 (GraphPad), luego se relacionaron las variables frente a los resultados obtenidos y se realizó el análisis estadístico en el programa SPSS versión 15 (SPSS Inc., Chicago, USA). Un primer análisis descriptivo se realizó con el total de la muestra, se calculó la prevalencia de sífilis en gestantes, así como las distribuciones de frecuencia para las diferentes variables. A través de un análisis bivariado, la definición de variables cualitativas independientes significativas se realizó con la distribución exacta de Fisher para comparar las razones de chance para cada desenlace comparado. Como medida de incertidumbre y significación estadística se tomó en cuenta el cálculo del intervalo de confianza.

\section{RESULTADOS}

El total de mujeres que se incluyeron en el estudio fueron 101 gestantes. La edad promedio de todo el grupo estudiado fue de 24 años (DE: \pm 6 años; rango entre los 15 y 47 años), la edad gestacional promedio fue de 20 semanas (DE: \pm 8 semanas). La descripción de acuerdo al estado civil de la muestra estudiada es la siguiente: $11 \%$ estaban casadas, 38\% convivían en unión libre, $32 \%$ eran solteras y del 19\% restante no se logró conseguir datos en este aspecto. La mayoría de las pacientes $(80 \%)$ se encontraban en el régimen subsidiado, el $4 \%$ pertenecían al régimen contributivo y el 3\% no estaban vinculadas al Sistema General de Seguridad Social; para el 17\% restante no fue posible establecer la información.

Se encontró que el 17\% de mujeres de la muestra estudiada no asistían al programa de atención integral a la gestante (control prenatal), el cual en Colombia -según la norma 412 del Ministerio de Salud y Protección Social- "debe ser mensual hasta la semana 36, y luego cada 15 días hasta la semana 40" y en "el último mes de gestación (semanas 36, 38 y 40) deben ser realizados por un médico". El 5\% de las mujeres 
-corroborado por la revisión a las historias clínicas- presentaban un antecedente de haber sufrido una enfermedad de transmisión sexual. Al momento de ser captadas, se encontró al examen físico: dos casos de condilomas, dos casos de secreciones purulentas que fueron diagnosticadas etiológicamente como gonorrea, y un caso de lesiones compatibles con herpes genital.

Finalmente, con el programa de demanda inducida (i.e. la búsqueda de casos por fuera de la oferta de servicios sanitarios) se lograron identificar 3 casos de sífilis gestacional (2,97\%), definidas como reactivas en las pruebas no treponémicas (VDRL). La Tabla 1 resume las principales características de las mujeres reactivas halladas a lo largo del estudio.

Se establecieron algunas asociaciones estadísticas como cálculo de razones de chance (Odds Ratio), comparación de tasas de incidencia, razón de las mismas y factores de riesgo, sobre todo con el propósito de generar hipótesis futuras que serán objeto de demostración en trabajos futuros y con metodologías idóneas para ello. Teniendo en cuenta esta advertencia metodológica, se estableció que tener un antecedente de sífilis aumenta el riesgo de sufrir sífilis gestacional (OR: 2,8 - IC95: 1,8-7,2).

Tabla 1. Casos positivos para sífilis gestacional.

\begin{tabular}{cccccccc}
\hline Casos & $\begin{array}{c}\text { Edad } \\
\text { (años) }\end{array}$ & Estado civil & $\begin{array}{c}\text { Edad } \\
\text { gestacional } \\
\text { (semanas) }\end{array}$ & $\begin{array}{c}\text { Afiliación a } \\
\text { Seguridad } \\
\text { Social }\end{array}$ & $\begin{array}{c}\text { Asiste a } \\
\text { control } \\
\text { prenatal }\end{array}$ & $\begin{array}{c}\text { Historia } \\
\text { de ITS }\end{array}$ & $\begin{array}{c}\text { Resultados } \\
\text { pruebas FTA }\end{array}$ \\
\hline 1 & 19 & Soltera & 8 & Subsidiado & No & No & Positivo \\
2 & 18 & Unión libre & 24 & Subsidiado & No & No & No se realizó \\
3 & 31 & Unión libre & 32 & Subsidiado & Sí & Ś́filis & Positivo \\
\hline
\end{tabular}

\section{DISCUSIÓN}

El estudio ha logrado dimensionar una grave problemática en dos comunidades vulnerables, en las cuales se hallaron tres mujeres con VDRL reactivo, una se encontraba en situación de privación de la libertad en la Cárcel de Mujeres La Badea, las otras dos no, sin embargo, una de ellas lo había estado en el pasado.

Curiosamente, estas tres mujeres que resultaron positivas estaban por fuera de la cobertura de las instituciones socialmente responsables del ingreso y seguimiento de las gestantes, siendo bastante compleja la localización de los contactos sexuales, situación que tiene repercusiones importantes en la salud pública dadas por el riesgo de transmisión, reinfección e infección en la comunidad en general, denotando a su vez la dificultad que aún tienen todos los actores del sistema para vigilar, controlar e intervenir un problema de interés en salud pública.

Es importante insistir en la importancia del hallazgo de gestantes positivas por fuera de los programas de control prenatal. La cobertura universal en salud -el concepto más poderoso que tiene la Salud Pública (31)- debe priorizar las enfermedades y riesgos que tengan las mujeres en embarazo y la primera infancia. Vale la pena entonces plantear la cuestión sobre lo que se puede inferir de esta omisión: ¿Las instituciones tienen una capacidad limitada para identificar y garantizar el tratamiento oportuno de calidad para las poblaciones vulnerables; o estas tienen pocos incentivos para buscar la atención del sistema de salud? 
Los presentes hallazgos van en concordancia con lo reportado por Galban y Benzaken, que demuestran la tendencia al aumento de la sífilis congénita en 9 países de LAC, incluido Colombia (26). De igual manera, García et al. en su estudio señalan que el $15 \%$ de las gestantes manifestaron inasistencia al control prenatal, y en el presente estudio el $17 \%$ de mujeres de la muestra estudiada no asistían a un programa de atención integral a la gestante (control prenatal), lo cual supone un importante factor de riesgo materno y perinatal, pues es el único medio que permite detectar e intervenir oportunamente alteraciones durante la gestación que en muchos de los casos no son percibidas por las madres (32).

Al observar de forma más amplia en Ceará (Brasil), Chaves et al. (33) presentan un estudio sobre sífilis congénita realizando un análisis epidemiológico de una década en dicha población. La edad de las mujeres embarazadas con sífilis de Ceará oscila entre 20 y 34 años, y en el presente estudio la edad promedio fue de 24 años. Estos resultados explican que el pico de la fase reproductiva es el más susceptible para adquirir este tipo de infecciones; es importante anotar -y esto será objeto de estudio en investigaciones posteriores- que esta edad sea precisamente donde se presenta el mayor número de embarazos, es decir, de mujeres posiblemente expuestas a sufrir el desenlace de interés (sífilis gestacional) (33).

En tanto, para la prevención de la sífilis congénita es necesaria una mayor concientización -en todos los niveles de atención- acerca del alcance y la gravedad de esta (34), especialmente en mujeres embarazadas y niños, garantizando la continuidad de programas a largo plazo donde se realice una búsqueda activa de la sífilis y otras ITS, para evitar la subnotificación y el subregistro $(35,36)$, propendiendo por el logro de la meta planteada por la Organización Panamericana de la Salud de 0,5 casos de SC por cada 1.000 nacidos vivos, incluyendo muertes fetales $(7,8$, 20). De la misma manera, se debe informar sobre la enfermedad a los usuarios de los servicios en comunidades vulnerables, convenciéndolos de la importancia de la prevención y el tratamiento para aportar ventajas sustanciales en la salud del binomio madre-hijo.

Es importante señalar que, mientras la sífilis tenga alta incidencia en la población adulta, la probabilidad de transmisión congénita seguirá siendo muy alta. Se observó en la población de estudio un amplio sometimiento decisorio con el compañero sexual, factor que influyó de forma significativa en la negativa a la participación del estudio. Por tal motivo, se deben adoptar medidas de educación en salud reproductiva y sexual tanto para la gestante como para su pareja, aplicando un enfoque integrado de atención maternoinfantil basado en el derecho a la información, asesoramiento y confidencialidad (37).

Sin embargo, otros estudios de LAC refieren menores prevalencias de SG que las del presente estudio, hallando que la condición topográfica del sujeto de estudio se encuentra directamente relacionada con la SG, evidenciando que en Perú, si bien la prevalencia general es del $0,7 \%$-con una población de 526.452 gestantes-, se reportan cifras de 1,3\% para la región selvática, $0,5 \%$ para la región costera y $0,5 \%$ para la sierra, donde los principales factores asociados a la positividad de la prueba de sífilis son ausencia de educación y bajo número de controles prenatales, mayor paridad y soltería, antecedente de recién nacido con bajo peso al nacer y vivir en la región selvática (38).

La aparición de casos de SC pone de manifiesto deficiencias de orden estructural y técnico en los servicios de salud, situación ante la cual se hace necesario instaurar una consulta preconcepcional con insistencia en la prevención de ITS, así como su tamizaje, tratamiento, búsqueda de contactos sexuales y seguimiento de los mismos. De esta forma, aumentar la demanda y la calidad del control prenatal, en términos de cantidad y tiempo por consulta, se configura como un aspecto de igual importancia que lo enunciado previamente. 
La prevalencia del estudio revela que la SG sigue siendo frecuente y aumenta la probabilidad de sufrir consecuencias nefastas para la unidad familiar, la comunidad y el sistema de salud en general. Es importante resaltar el hecho de que el antecedente de sífilis esté asociado con la sífilis gestacional: esto estaría hablando de una falla terapéutica, las causas de esta serán objeto de estudios posteriores. Finalmente, es importante concluir que el diagnóstico de sífilis, en este caso, estuvo acompañado de otras ITS por lo que cada caso debe ser revisado de forma extensa y profunda en búsqueda de otras comorbilidades.

\section{AGRADECIMIENTOS}

ARicardo de Jesús Jiménez-Mejía, Rector ejecutivo de la Fundación Universitaria Autónoma de las Américas y Decano de la Facultad de Medicina, por su incondicional apoyo logístico y administrativo. A Catalina López-Salazar por sus traducciones. A la Fundación Universitaria Autónoma de las Américas y al Hospital Santa Mónica de Dosquebradas por su apoyo, financiamiento y su propuesta de promoción de la salud y prevención de la enfermedad en las comunidades vulnerables de la región.

\section{FINANCIACIÓN}

La investigación fue financiada por la Fundación Universitaria Autónoma de las Américas y el Hospital Santa Mónica de Dosquebradas.

\section{CONFLICTO DE INTERESES}

Los autores declaran no tener ningún conflicto de intereses.

\section{REFERENCIAS}

1. World Health Organization. Global Prevalence and Incidence of Selected Curable Sexually Transmitted Infections Overview and Estimates. Geneva; 2011.

2. Blencowe $H$, Cousens $S$, Kamb M, Berman S, Lawn JE. Lives saved tool supplement detection and treatment of syphilis in pregnancy to reduce syphilis related stillbirths and neonatal mortality. BMC Public Health 2011; 11(3):1-16.

3. Mattei PL, Beachkofsky TM, Gilson RT, Wisco OJ. Syphilis: A Reemerging Infection. Am Fam Physician 2012; 86(5):433-440.

4. Galeano CL, García WD, Congote LM, Vélez MA, Martínez DM. Prevalencia de sífilis gestacional e incidencia de sífilis congénita, Cali, Colombia. Rev Colomb Obstet Ginecol 2010; 63(4):321-326.

5. Instituto Nacional de Salud. Referencia en Salud Pública. Manual para el Diagnóstico de Sífilis 2011. CÓDIGO: MNL-R01.001.5030-013.

6. French P. Syphilis. BMJ 2007; 334:143-147.

7. Arnesen L, Martínez G, Mainero L, Serruya S, Durán P. Gestational syphilis and stillbirth in Latin America and the Caribbean. Int J Gynaecol Obstet 2015; 128(3):241-5.

8. Bradley H, Tapia V, Kamb ML, Newman LM, García PJ, Serruya SJ, et al. Can the Perinatal Information System in Peru be used to measure the proportion of adverse birth outcomes attributable to maternal syphilis infection? Rev Panam Salud Pública 2014; 36(2):73-9.

9. Cruz AR. Situación de la Sífilis gestacional y congénita en Colombia, un desafío al Sistema General de Seguridad Social en Salud. Rev Colomb Obstet Ginecol 2012; 63(4):304-306.

10. Kamb ML, Newman LM, Riley PL, Mark J, Hawkes SJ, Malik T, et al. A Road Map for the Global Elimination of Congenital Syphilis. Obstetrics and Gynecology International 2010; 1-6. 
11. Schmid GP, Stoner BP, Hawkes S, Broutet N. The Need and Plan for Global Elimination of Congenital Syphilis. Sexually Transmitted Diseases 2007; 34(7):5-10.

12. Magalhães DM, Laudares IA, Dias A, Paranhos I. Maternal and Congenital Syphilis: A persistent challenge. Cad Saude Pública 2013; 29(6):1109-1120.

13. Díaz LA. Sífilis gestacional: Un problema de salud pública. Rev Fac Med 2011; 59(3):163-165.

14. Workowski KA, Bolan G. Sexually Transmitted Diseases Treatment Guidelines, 2014. Centers for Disease Control and Prevention; 2014.

15. Ministerio de Salud y Protección Social, Fondo de Población de las Naciones Unidas. Guía de Práctica Clínica basada en la evidencia para la atención integral de la Sífilis Gestacional y Congénita. Colombia; 2014.

16. Centers for Disease Control and Prevention. Morbidity and Mortality Weekly Report. Sexually Transmitted Diseases. Treatment Guidelines, 2010.

17. Lago EG, Vaccari A, Fiori RM. Clinical features and follow-up of congenital syphilis. Sex Transm Dis 2013; 40(2):85-94.

18. Newman L, Kamb M, Hawkes S, Gómez G, Say L, Seuc A, et al. Global estimates of syphilis in pregnancy and associated adverse outcomes: analysis of multinational antenatal surveillance data. PLoS Med 2013; 10(2):10.

19. OMS, OPS, Unicef. Recomendaciones Técnicas para la elaboración de protocolos para Estudios de Prevalencia de Sífilis y VIH en parteras y/o puérperas. Iniciativa para la eliminación de la transmisión maternoinfantil del VIH y de la Sífilis congénita en América Latina y el Caribe 2011. Publicación CLAP/ SMR No 1581.

20. Organización Panamericana de la Salud, Organización Mundial de la Salud. Estrategia y plan de acción para la eliminación de la transmisión maternoinfantil del VIH y de la sífilis congénita. Tercera Edición. Washington, D.C.; 2014.

21. Ministerio de la Protección Social. Dirección General de Salud Pública. Plan estratégico para la eliminación de la transmisión materno-infantil del VIH y de la sífilis congénita. Colombia 2011-2015.

22. Center for Diseases Control and Prevention. California Department of Public Health. Syphilis Elimination Effort Strategic Plan, 2011-2015.

23. Ministerio de Salud y Protección Social, Fondo de Población de las Naciones Unidas. Guía de Práctica Clínica Basada en la Evidencia para la Atención Integral de la Sífilis Gestacional y Congénita 2014. Colombia.

24. Pan American Health Organization. Guidance on Syphilis Testing in Latin America and the Caribbean: Improving Uptake, Interpretation, and Quality of Testing in Different Clinical Settings. Washington, DC: PAHO; 2015.

25. Serruya SJ, Durán P, Martínez G, Romero M, Caffe S, Alonso M, et al. Maternal and congenital syphilis in selected Latin America and Caribbean countries: a multi-country analysis using data from the Perinatal Information System. Sex Health 2015; 12(2):164-169.

26. Galban E, Benzaken A. Situación de la sífilis en 20 países de Latinoamérica y el Caribe: año 2006. DST - J bras Doenças Sex Transm 2007; 19(3-4):166-172.

27. INS, SIVIGILA. Informe del evento sífilis gestacional y congénita hasta el periodo epidemiológico 52 del año 2013.

28. Análisis de la situación de salud de Risaralda. Primer semestre de 2012. [Internet]. Disponible en: http://www.minsalud.gov.co/plandecenal/mapa/Analisis-de-Situacion-Salud-Risaralda-I-2012.pdf. Consultado septiembre de 2012.

29. WIENER. Suspensión antigénica estabilizada para realizar la prueba VDRL modificada (USR) de detección de sífilis. [Internet]. Disponible en: http://www.wiener-lab.com.ar/VademecumDocumentos/ Vademecum\%20espanol/vdrl_test_sp.pdf. Consultado octubre de 2013. 
30. George RW, Hunter EF, Fears M. Fluorescent Treponemal Antibody-Absorption (FTA-ABS) Test. Chapter 12. [Internet]. Disponible en: http://www.cdc.gov/std/syphilis/manual-1998/CHAPT12. pdf. Consultado enero de 2015.

31. OMS. Investigación para la cobertura universal de salud: Informe Mundial de Salud 2013. Disponible en: http://www.who.int/whr/2013/report/en/

32. García LM, Almanza RA, Miranda C, Gaviria JM, Julio JM, Paternina ME, et al. Factores de riesgo asociados a la sífilis congénita en tres instituciones de salud de Sincelejo (Colombia). Dialnet 2011; $8(1): 34-41$.

33. Chaves C, Freitas LV, Do Nascimento DM, De Oliveira LL, Araujo ACM, De Oliveiria MV, et al. Sífilis congénita en Ceará: análisis epidemiológico de una década. Brasil. Rev Esc Enferm USP 2013; 47(1):152-9.

34. Hawkes S, Matin N, Broutet N, Low N. Effectiveness of interventions to improve screening for syphilis in pregnancy: a systematic review and meta-analysis. The Lancet Infectious Diseases $2011 ; 11(9): 684-$ 691.

35. Universidad Nacional de Colombia. Sífilis gestacional: un problema de salud pública. Rev Fac Med Univ Nac Colomb 2011; 59(3):163-165.

36. Organización Panamericana de la Salud, Organización Mundial de la Salud, Programa Conjunto de las Naciones Unidas sobre el VIH/SIDA. Pautas para la vigilancia de infecciones de transmisión sexual. Programa Regional de Sida de la Organización Panamericana de la Salud; 1999.

37. Organización Mundial de la Salud. Catalogación por la biblioteca de la OMS: Eliminación mundial de la sífilis congénita: fundamentos y estrategia para la acción; 2008.

38. Gonzales GF, Tapia V, Serruya SJ. Gestational syphilis and associated factors in public hospitals of Peru during 2000-2010. Rev Peru Med Exp Salud Pública 2014; 31(2):211-21. 